\title{
Safety of Live Retrograde Intrarenal Surgery; Results from a Boutique Course Series
}

\author{
Oktay Ozman ${ }^{1}$, Sinharib Citgez ${ }^{2}$, Cem Basatac $^{1}$, Murat Akgul ${ }^{3}$, Cenk Murat Yazıci ${ }^{3}$, \\ Yiloren Tanidir ${ }^{4}$, Haluk Akpinar ${ }^{1}$, and Bulent Onal ${ }^{5}$ \\ ${ }^{1}$ Affiliation not available \\ ${ }^{2}$ Istanbul University-Cerrahpasa Cerrahpasa Faculty of Medicine \\ ${ }^{3}$ Namik Kemal University Faculty of Medicine \\ ${ }^{4}$ Marmara University School of Medicine \\ ${ }^{5}$ Istanbul Universitesi-Cerrahpasa
}

November 29, 2020

\begin{abstract}
Introduction: This study aims to investigate the outcomes and complication rates of patients undergoing retrograde intrarenal surgery (RIRS) at the live surgery events organized as boutique course series. Materials and Methods: Eight RIRS courses were organized between November 2017 and February 2020. Data of 24 patients who were operated in the live surgery events (as LSE group) for renal stone were matched with the data of 24 substitute patients (as control group) who underwent regular RIRS on the same period at the same centers.. Results: Stone free status of groups was similar (88\% in LSE and $79 \%$ in the control group; $\mathrm{p}=1$ ). There was no significant difference in terms of complication and need for additional procedure rates, operation and fluoroscopy and hospitality times between the two groups ( $\mathrm{p}=1, \mathrm{p}=1, \mathrm{p}=0.12, \mathrm{p}=0.58$ and $\mathrm{p}=0.94$, respectively). Fifty-four \% (13/24) of LSE operations were performed by guest surgeons. No statistically significant difference was found between the patients who operated by host and guest surgeons. However, the operation times of the operations performed by guest surgeons were longer than those performed by the host surgeons ( $96.5 \pm 28$ and $66.5 \pm 30$ minute, respectively, $\mathrm{p}=0.07$ ). Conclusion: Our study is the first report on this area. RIRS live surgery can be performed with low complication and high stone-free rates without jeopardizing patient safety. If the surgeon is not familiar with the operating room set-up or staffs, the live surgery must performed by the host surgeon to avoid extended operating time.
\end{abstract}

\section{Introduction}

Kidney stones are an important health problem with a high prevalence worldwide. According to recent studies, the prevalence of kidney stones in the United States was found to be $8.8 \%$ (1). This rate is between $1 \%$ and $15 \%$ worldwide, depending on age, gender, race and geographical area (2).

The frequent occurrence of kidney stones have increased the need for more effective and safe methods in stone treatment. Advances in fiberoptic technologies, intracorporeal lithotripters and grasping devices have made retrograde intrarenal surgery (RIRS) an effective and safe minimally invasive method for treatment (3).

The European Association of Urology (EAU) Guidelines recommends the RIRS as an alternative approach when the percutaneous nephrolithotomy and shock wave lithotripsy (SWL) are not an option even for larger than $2 \mathrm{~cm}$ in size (4). Retrograde intrarenal surgery is a sophisticated surgical procedure that requires the efficient use of different technologies at the same time. Surgical experience is an important factor affecting the safety of RIRS (5). Live surgery events (LSEs) are frequently organized to transfer surgical experiences 
to surgeons beginning of the learning curve. On the other hand, live surgery is a controversial issue in terms of ethics and legal (6). The European Association of Urology publishes its policy on live surgical activities with a review of panel recommendations (7). But previous studies do not provide a direct evidence of higher complication and lower stone-free rates that can make RIRS an ethic and legal problem in LSEs.

This study aims to investigate the outcomes and complication rates of patients undergoing RIRS at the LSEs organized by the Society of Urological Surgery in Turkey (SUST) and compare it with a matched control group.

\section{Material and Methods}

Eight RIRS courses were organized by SUST between November 2017 and February 2020. Data of 24 patients who underwent live RIRS (as LSE group) were consecutively enrolled to the study. The control group were selected from patients who underwent regular RIRS on the same period at the same centers. Surgeon and stone burden matched 24 control cases included to the study by making one-to-one matching. All procedures were performed in four different centers which has high institutional experience in endourology field. Written informed consent was obtained from all patients before the procedure. Patients who will undergo live surgery were selected among those who were previously indicated for RIRS and the operation was planned. Informed consent was specifically state that the surgery was going to be viewed by course participants for live surgery cases.

In the LSE group, the trainees watched operations directly in the operation room, taking care of the sterile field. Four or 5 participant were present in the operating room during the cases. Surgeons performing the procedure interacted with the trainees and the details of the operation were explained during the live cases by the surgeon. Also there was a moderator to field questions in the operating room. The moderator also acted as the patient's advocate. Radiation protection precautions were taken for all trainees.

Live surgery cases were performed by 5 different surgeons. All of them had advanced RIRS experience (each surgeon performed at least 100 RIRS procedures). All surgeons had previously participated in different LSEs. If a guest surgeon performed the case, he was assisted by his regular resident but not regular staff nurse. The same equipment, instruments and devices which had been used by all surgeons previously were moved to host institutes for each course. Surgeon's familiarity status with all external factors is shown in Table 1. Flex-X2 ${ }^{\circledR}$ (Karl Storz Endoscope), URF-P5 ${ }^{\circledR}$ (Olympus) flexible URS and URF-V ${ }^{\circledR}$ (Olympus) were used in procedures. All cases were digitally recorded. Control cases were operated by the same 5 surgeons in their hospitals. Ureteral access sheath was used in all of patients. Lithotripsy was done with Holmium laser lithotripters from various companies (Stonelight $30^{\circledR}$, Quanta System Litho ${ }^{\circledR}$ ). Surgical prophylaxis was given with ceftriaxone (i.v.) before the operation.

Preoperative characteristics, perioperative and postoperative findings of the LSE group were recorded and compared with the control group. The patients were routinely evaluated with hemogram, routine biochemical parameters, urinalysis and urine culture before the procedure. All patients were evaluated by non-contrast low-dose computed tomography $(\mathrm{CT})$ before the procedure and the Hounsfield Unit (HU) of stones were measured. Stone free status was evaluated perioperatively by using fluoroscopy and in the postoperative first month by using non-contrast low-dose CT. Perioperative and postoperative complications were classified according to the Clavien and Satava Classifications respectively (8-10). Based on the results of the procedure, the patients were evaluated as stone-free or as having residual stone (any evidence of persistent stone fragments irrespective of size). Besides, LSE patients were divided into two subgroups according to the surgeon's status (host or guest surgeon) and the results were compared with each other. Stone-free results and all complications from live surgery cases has been presented back to the participants by e-mail.

The Chi-Square and Fisher exact tests were used for the difference between the categorical variables. MannWhitney U test was used for the difference between the means. The data were analyzed with the Statistical Package for the Social Sciences v. 22 (SPSS Inc, Illinois, USA). A p-value under 0.05 was considered statistically significant. 


\section{Results}

The mean age of the patients in LSE and control groups was 45.0 \pm 15.6 (median 45) and 42.5 \pm 18.7 (median $44)$, respectively and there was no statistically significant difference between the two groups $(\mathrm{p}=0.71)$. In addition, we did not find statistical significance between the two groups in terms of gender, side, previous history of failed SWL for same stone, maximum stone diameter, stone density, stone locations, and preoperative double $\mathrm{J}$ stent placement rates $(\mathrm{p}=0.77, \mathrm{p}=0.37, \mathrm{p}=0.77, \mathrm{p}=0.21, \mathrm{p}=0.72, \mathrm{p}=0.29$, and $\mathrm{p}=0.76$, respectively). (Table 2 )

Mean operation and fluoroscopy times were statistically indifferent to both groups $(\mathrm{p}=0.12, \mathrm{p}=0.58$ respectively). Stone free status of groups was similar $(88 \%$ in LSE and $79 \%$ in the control group; $\mathrm{p}=0.33$ ). There was no difference in complication rates between the two groups also ( $17 \%$ vs $13 \%, \mathrm{p}=0.9)$. (Table 3 )

Mild bleeding was observed in one patient in the LSE group, and minimal mucosal injury was observed in one patient in both groups perioperatively. Ureteral access could not be provided in one LSE patient and SWL was planned for a different session. Mucosal injury requiring stent insertion occurred in one patient from the control group. Two patients had fewer (one in LSE group which is requiring antipyretics and one in the control group which is requiring antibiotics). One patient had acute urine retention after double $\mathrm{J}$ stent removal (as Grade 2 complication in the control group). One patient developing renal colic for ureteral residual stone fragment underwent ureterorenoscopy (as Grade 3 complication in LSE group). All complications presented in Table 4 according to modified Satava and Clavien Classifications.

Fifty-four \% (13/24) of LSE operations were performed by guest surgeons. It was the first time working with the staff in the OR for these surgeons. Perioperative and postoperative outcomes of the LSE group according to surgeons status are shown in Table 5. No statistically significant difference was found between the two groups in any parameter.

\section{Discussion}

Live surgical events create unique learning opportunities for participants. SUST organized RIRS courses in different clinics between 2017-2020. Through these courses, close to 100 urologists have had the chance to learn the tips and tricks of RIRS by closely watching from experienced endourologists. In the present study, we report that all sessions were performed with satisfaction and there were no major complications during the live surgeries.

Despite the positive results in terms of educators and participants, live surgical activities bring about some ethical and legal debates from the patient's point of view. Although the vast majority of respondents in a survey on the American Association of Genitourinary Surgeons found that live surgical activity was ethical, just $28.2 \%$ of them could allow themselves or their relatives to undergo surgery in such an event (11). It is argued that surgeons working under greater stress than regular surgery may cause potential hazards for the patient (12). In a study of surgeons reporting personal experience of LSE; $6.5 \%$ of respondents noted 'significant anxiety' increasing to $19.4 \%$ when performing surgery away from home (13). It has been reported that more staff in the operating room during live surgical events may increase the risk of surgical site infection (14). But the size of the event can also affect the surgeon's anxiety level. Live surgery during a course with a few course participants in the room does not cause the same level of stress/anxiety to the surgeon as live surgery during a large online or live event at a conference with often more than a thousand people watching the procedure. In our courses there were no more people than during regular education or training activity. Boutique live surgeries can eliminate ethical concerns in this area. Because, this is especially common in medical faculty clinics for training medical students and residents. Live surgeries are a requirement for effective medical education and postgraduate training. Our courses have been conducted in large centers with experienced staff who can manage the stress and the risks of event in such trainings.

Urological studies investigating LSE safety are limited. As expected, robotic surgery using state-of-the-art technologies provides the best data on this issue. In a study in which the results of 39 robotic partial nephrectomies were reported, patient outcomes were found to be similar to normal operating procedure (15). 
Similar results have been reported in another single-center study in which data from 36 robot assisted radical prostatectomy cases were included (12). The results of patients who underwent GreenLEP in live surgery events reported by Misrai et al. were compared with regular surgery results. The author emphasized that such practices do not endanger the skill and technique of the surgeon (16). The largest-scale study in this area has recently been reported by Rocco et al. (17). The overall rate of complications in laparoscopic and robotic surgery performed by 27 different surgeons was $11.6 \%$. It was concluded that complication rates were low due to the difficulty of serially operated operations such as radical prostatectomy and cystectomy. To the best of our knowledge, there are no live surgery studies reported in the literature on RIRS. In this study, we found similar stone-free and complication rates for LSE's and regular RIRS cases.

European Association of Urology (EAU) policy on live surgery events has been presented at the 28th Annual EAU Congress on behalf of the EAU Live Surgery Committee (EAU-LSC) (18). Accordingly, the association accepts the use of live surgery as an academic technique. The report concluded patient safety as the most important principle. In our study complication rates of live surgery patients similar to regular surgery patients.

Surgeons tend to stay away from extremes and choose standard cases in live surgical events. The EAU also recommends the selection of standard cases as much as possible so that the educational objectives are not overlooked. The preoperative characteristics of the cases in our series are summarized in Table 2 and it was similar to those of regular surgery performed. Some patients who have double J stent and unsuccessful SWL history were selected specifically. SWL-resistant kidney stones constitute a large area of indications for RIRS. It is also easier to access patients with ureteral double J stent prior to RIRS. The facts with the above features are especially preferred because it is an important part of the education. The slightly longer duration of the LSE cases can be attributed to the trainees being explained each level during the operation. Another reason could be guest surgeons unfamiliarity with the operating room set-up or staff (except assisting surgeon) The duration of any operation did not exceed 120 minutes except for a case that had an ipsilateral open ureterolithotomy history and a stone in the caliceal diverticulum.

We compared the outcomes and complication rates of patients undergoing live RIRS with the matched control group. We did not find a statistically significant difference between these groups according to the stone-free status and complication rates. Complication rates with RIRS are generally low. The number of patients in the study was not sufficient to obtain statistically significant differences between the complications and equivalency in other continuous clinical parameters. That was the main limitation of the study. Another limitation of our study, was that the surgeries were performed by five different surgeons (as host or guest). However, the results did not change according to the host or guest surgeons. We believe that organizing the same course with the same surgical team has a positive effect on live surgery courses. This is an important factor that minimizes surgeon anxiety, although we do not use an anxiety scale to compare the two groups.

\section{Conclusion}

RIRS, supported by new technologies, is implemented more frequently in everyday practice because of the increasing incidence of kidney stones. As a result, surgical education on RIRS have become more important. Our study presents data on this area. RIRS live surgery can be performed with low complication and high stone-free rates without jeopardizing patient safety. If the surgeon is not familiar with the operating room set-up or staffs, the live surgery must performed by the host surgeon to avoid extended operating time.

\section{References}

1. Scales CD, Jr., Smith AC, Hanley JM, et al. Prevalence of kidney stones in the United States. Eur Urol. 2012;62(1):160-5.

2. Romero V, Akpinar H, Assimos DG. Kidney stones: a global picture of prevalence, incidence, and associated risk factors. Reviews in urology. 2010;12(2-3):e86-96.

3. de la Rosette J, Denstedt J, Geavlete P, et al. The clinical research office of the endourological society ureteroscopy global study: indications, complications, and outcomes in 11,885 patients. J Endourol. 
2014;28(2):131-9.

4. Turk C, Petrik A, Sarica K, et al. EAU Guidelines on Interventional Treatment for Urolithiasis. Eur Urol. 2016;69(3):475-82.

5. Berardinelli F, Cindolo L, De Francesco P, et al. The surgical experience influences the safety of retrograde intrarenal surgery for kidney stones: a propensity score analysis. Urolithiasis. 2017;45(4):387-92.

6. Cumpanas AA, Ferician OC, Latcu SC, et al. Ethical, legal and clinical aspects of live surgery in urology - contemporary issues and a glimpse of the future. Wideochirurgia $\mathrm{i}$ inne techniki maloinwazyjne $=$ Videosurgery and other miniinvasive techniques. 2017;12(1):1-6.

7. Collins JW, Akre O, Wiklund PN. Re: Walter Artibani, Vincenzo Ficarra, Ben J. Challacombe, et al. EAU policy on live surgery events. Eur urol 2014; 66:87-97. Eur Urol.2014; 66(6):e121-2.

8. Dindo D, Demartines N, Clavien PA. Classification of surgical complications: a new proposal with evaluation in a cohort of 6336 patients and results of a survey. Annals of surgery. 2004;240(2):205-13.

9. Oguz U, Resorlu B, Ozyuvali E, et al. A. Categorizing intraoperative complications of retrograde intrarenal surgery. Urol Int. 2014;92(2):164-8. doi: 10.1159/000354623. Epub 2014 Feb 6.

10. Xu Y, Min Z, Wan SP, et al. Complications of retrograde intrarenal surgery classified by the modified Clavien grading system. Urolithiasis. 2018 Apr;46(2):197-202. doi: 10.1007/s00240-017-0961-6. Epub 2017 Feb 24.

11. Duty B, Okhunov Z, Friedlander J, et al. Live surgical demonstrations: an old, but increasingly controversial practice. Urology. 2012;79(5):1185.e7-11.

12. Ogaya-Pinies G, Abdul-Muhsin H, Palayapalayam-Ganapathi H, et al. Safety of Live Robotic Surgery: Results from a Single Institution. Eur Urol Focus. 2019;5(4):693-697.

13. Khan SA, Chang RT, Ahmed K, et al. Live surgical education: a perspective from the surgeons who perform it. BJU Int. 2014;114(1):151-8.

14. Pryor F, Messmer PR. The effect of traffic patterns in the OR on surgical site infections. AORN journal. 1998;68(4):649-60.

15. Mullins JK, Borofsky MS, Allaf ME, et al. Live robotic surgery: are outcomes compromised? Urology. 2012;80(3):602-7.

16. Misrai V, Guillot-Tantay C, Pasquie M, et al. Comparison of Outcomes Obtained After Regular Surgery Versus Live Operative Surgical Cases: Single-centre Experience with Green Laser Enucleation of the Prostate. Eur Urol Focus. 2019;5(3):518-524.

17. Rocco B, Grasso AAC, De Lorenzis E, et al. Live surgery: highly educational or harmful? World J Urol. 2018;36(2):171-5.

18. Artibani W, Ficarra V, Challacombe BJ, et al. EAU policy on live surgery events. Eur Urol. 2014;66(1):8797.

Table 1. Surgeon's familiarity status with external factors

\begin{tabular}{llll}
\hline Characteristic & Regular Surgeries & LSE with Host Surgeon & LSE with Guest Surgeon \\
Instruments & + & + & + \\
OR set-up & + & + & $-/+$ \\
OR staff & + & + & - \\
Assisting surgeon/resident & + & + & + \\
Institution & + & + & $-/+$ \\
\hline
\end{tabular}


+ ; familiar, -/+ ; little familiar, - ; unfamiliar

Table 2. Patient Baseline Characteristics

\begin{tabular}{llll}
\hline Characteristic & LSE Group & Control Group & p value \\
Number & 24 & 24 & \\
Age (year, mean) & $45.0 \pm 15.6$ & $42.5 \pm 18.7$ & 0.71 \\
(median, range) & $45(2-72)$ & $44(5-66)$ & \\
Gender & & & \\
Male & $10 / 24(42 \%)$ & $12 / 24(50 \%)$ & 0.77 \\
Female & $14 / 24(58 \%)$ & $12 / 24(50 \%)$ & \\
BMI $\left(\mathrm{kg} / \mathrm{m}^{2}\right)$ & $27.2 \pm 3.2$ & $27.0 \pm 5.1$ & 0.57 \\
Side & & & \\
Right & $13 / 24(54 \%)$ & $17 / 24(71 \%)$ & 0.37 \\
Left & $11 / 24(46 \%)$ & $7 / 24(29 \%)$ & \\
Stone Burden $(\mathrm{mm})$ & $11.9 \pm 3.1$ & $11.1 \pm 4.2$ & 0.21 \\
Hounsfield Unit (mean) & $1100 \pm 377$ & $1076 \pm 358$ & 0.72 \\
Localisation & & & \\
Lower Calyx & $4 / 24(17 \%)$ & $4 / 24(16 \%)$ & \\
Other Calyces & $5 / 24(21 \%)$ & $9 / 24(38 \%)$ & 0.29 \\
Pelvis & $14 / 24(58 \%)$ & $8 / 24(33 \%)$ & \\
Multiple Loc. & $1 / 24(4 \%)$ & $3 / 24(13 \%)$ & \\
Previous history of failed SWL for same stone & $12 / 24(50 \%)$ & $13 / 24(58 \%)$ & 0.77 \\
Preoperative Double J Stent & $9 / 24(38 \%)$ & $8 / 24(33 \%)$ & 0.76 \\
\hline
\end{tabular}

BMI: Body Mass Index

Table 3. Perioperative and Postoperative Outcomes

\begin{tabular}{llll}
\hline Characteristic & LSE Group & Control Group & p value \\
Operation Time (minute) & $82.2 \pm 31$ & $73.8 \pm 21$ & 0.12 \\
Floroscopy Time (second) & $23.6 \pm 21$ & $15.7 \pm 9$ & 0.58 \\
Length of Hospitality (day) & $1.8 \pm 1$ & $1.9 \pm 2$ & 0.94 \\
Stone Free Rate & $21 / 24(88 \%)$ & $19 / 24(79 \%)$ & 1 \\
Complication Rate & $4 / 24(17 \%)$ & $3 / 24(13 \%)$ & 1 \\
Need for Additional Procedure & $2 / 24(8 \%)$ & $1 / 24(4 \%)$ & 1 \\
Postoperative Double J Stent & $23 / 24(96 \%)$ & $23 / 24(96 \%)$ & 1 \\
\hline
\end{tabular}

Table 4. Complication characteristics

\begin{tabular}{llll}
\hline $\begin{array}{l}\text { Variable } \\
\begin{array}{l}\text { Postoperative } \\
\text { complications according }\end{array}\end{array}$ & $\begin{array}{l}\text { Grade } \\
\text { to the modified Clavien }\end{array}$ & $\begin{array}{l}\text { LSE Group } \\
1(50 \%)\end{array}$ & $\begin{array}{l}\text { Control Group } \\
0(0 \%)\end{array}$ \\
$\begin{array}{l}\text { Classification } \\
\text { ( }\end{array}$ & 2 & $0(0 \%)$ & \\
& 3 & $1(50 \%)$ & $2(100 \%)$ \\
Postoperative & 4 & $0(0 \%)$ & $0(0 \%)$ \\
complications & & $\mathbf{2 ( 8 . 5 \% )}$ & $0(0 \%)$ \\
& & $\mathbf{2}(\mathbf{7 . 5 \% )})$
\end{tabular}


Perioperative

1

$2(66 \%)$

$1(50 \%)$

complications according

to modified Satava

Classification

\section{Perioperative}

complications

Overall complications

(patients)

$2 \mathrm{~A}$

$2 \mathrm{~B}$

3
$1(33 \%)$

$0(0 \%)$

$0(0 \%)$

$3(12.7 \%)$

$4(17 \%)$
$1(50 \%)$

$0(0 \%)$

$0(0 \%)$

$2(7.5 \%)$

$3(13 \%)$

Table 5. Peri/Postoperative Outcomes of LSE Group According to Surgeons

Characteristic

Number

Operation Time (minute)

Floroscopy Time (second)

Length of Hospitality (day)

Stone Free Rate

Complication Rate

Need for Additional Procedure
Table 5. Peri/Postoperative Outcomes of LS

\section{Host surgeons}

11

$66.5 \pm 30$

$26.4 \pm 28$

$1.5 \pm 0.8$

$91 \%(10 / 11)$

$27 \%(3 / 11)$

$9 \%(1 / 11)$

\section{Hosted file}

Title Page.pdf available at https://authorea.com/users/375408/articles/495944-safety-oflive-retrograde-intrarenal-surgery-results-from-a-boutique-course-series 